\title{
Multiple SolitonSolutions for a (1+1)-dimensionalHirota-Satsuma shallow water wave equation UsingPainlevé-Bäcklund Transformation and the Simplified Hirota's Method
}

\author{
M. Y. Adamu \\ Mathematical Sciences Programme, AbubakarTafawaBalewa University, Bauchi, Nigeria
}

\begin{abstract}
The multiple solitonsolutions of (1+1)-dimensional Hirota-Satsuma shallow water wave equation is studied usingPainlevé- Bäcklundtransformation and the simplified Hirota's method.Also the hyperbolic and theExp-trigonometric function methods are used to obtain some more kind of solitary wave solutions.
\end{abstract}

Keywords:Hirota Satsuma water wave equation, multiplesoliton solution, SimplifiedHirota's method, hyperbolic and Exp-trignometric solutions.

\section{Introduction}

The search for exact solution to non-linear differential equation is significantly important to mathematical physics. Exact solution plays vital roles in understanding various qualitative and quantitative feature of nonlinear phenomenon. There areclasses of interesting exact solutions such as solitonand the travelling wave solution, but it often needs for a specific mathematical technique to construct exact solution due to non-linearity property in dynamics $(\mathrm{Su}$, et al,2007)

Various methods have been employed to study integrable system and nonlinear evolution equations. Such as:Hirota bilinear method, (Hirota, 2004)the inverse scattering method,(Ablowitz, 1981)the Darboux transformation, the generalized symmetry method (Adamu and Suleiman, 2013),Wronskian determinant and etc (Ma, 2003).They all make it possible to create multiple soliton solutions for many integrable systems and nonlinear evolution equations. Yet, the Hirota's bilinear method and the simplified Hirota's method are more appealing to the solutions of the multiple soliton solutions for the nonlinear differential equation.(Hirota, 2004) We will in this workuse the idea proposed by (Hereman and Nuseir, 1997, Wazwaz, 2013) to investigate the $(1+1)$ - dimensionalHirota -Satsuma shallow water wave equation of the form:

$U_{x x t}+3 U U_{t}-3 U_{x} V_{t}-U_{x}=U_{t}$,

$V_{x}=-U$

for multiple soliton solution using the combine Painlëve-Bácklund transformation with simplified Hirota method and also to obtain travelling wave and singular solution using some hyperbolic and exp-trigonometric function methods. Equation (1) models the unidirectional propagation of shallow water wave, where $\mathrm{V}(\mathrm{x}, \mathrm{t})$ represents the horizontal velocity of water and $\mathrm{V}(\mathrm{x}, \mathrm{t})$ gives the deviation height from the equilibrium position of the liquid.(Hietaranta,,2009)

\section{Multiple soliton solutions}

According to (Wazwaz, 2013) we can definethe Paninlelvë - Bácklund transformation

$U(x, t)=(\operatorname{Inf})_{x}+U_{0}(x, t)$

$\left.V(x, t)=(\operatorname{Inf})_{x x}+V_{0}\right\}$

where $f$ is an auxiliary function ofx,t that will be used to get the one soliton solutions. The truncatedPainlevéexpression of the system (1) is used to obtain the transformation (2).

For the solution of (1) the function $U_{0}(x, t)$ and $V_{0}$ are considered as arbitraryansatz and for simplicity we set

$U_{0}=V_{0}=0$

which gives

$V(x, t)=U_{x}(x, t)$

Substituting (3) into (1) gives a single nonlinear equation

$2 U_{t}-3 U U_{t}-3 U_{x} U_{x t}+U_{x}=0$

In order to get the dispersion relation, we set 
$U(x, t)=(\operatorname{Inf}(x, t))_{x x}$

where the auxiliary function $f(x, t)$ is given by

$$
f(x, t)=1+e^{k_{i} x-c_{i} t}, i=1,2,3
$$

Substituting (5) in (4) gives the dispersion relation by

$$
C_{i}=\frac{k_{i}}{1-k_{i}^{2}}, \quad i=1,2,3
$$

Noting that the dispersion relation depends only on the coefficients of the variable $x$ Using (5) gives the kink and the soliton solutions

$$
\begin{aligned}
& U(x, t)=\frac{k_{1}^{2} e^{k_{i} x+\frac{k_{1} t}{k_{i}^{2}-1}}}{\left(1+e^{k_{i} x+\frac{k_{i} t}{k_{i}^{2}-1}}\right)^{2}} \\
& V(x, t)=\frac{k_{1}^{3} e^{k_{i} x+\frac{k_{1} t}{k_{i}^{2}-1}}\left(1+e^{k_{1} x+\frac{k_{1} t}{k_{i}^{2}-1}}\right)}{\left(1+e^{k_{i} x+\frac{k_{i} t}{k_{1}^{2}-1}}\right)^{3}}
\end{aligned}
$$

Respectively, where we used $V(x, t)=U_{x}(x, t)$ as given in (3)

For the two solitary wave solution we set the auxiliary function as

$$
f(x, t)=1+e^{\left(k_{1} x+\frac{k_{1} t}{k_{1}^{2}-1}\right)}+e^{\left(k_{2} x+\frac{k_{2} t}{k_{2}^{2}-1}\right)}
$$

Now substituting (9) and (5) into our equation (1) we obtain the two kink solutionsand the two soliton solutions by

$$
\begin{aligned}
& U(x, t)=\frac{k_{1}^{2} e^{\left(2 k_{1} x+\frac{2 k_{1} t}{k_{1}^{2}-1}\right)}+k_{2}^{2} e^{\left(2 k_{2} x+\frac{2 k_{2} t}{k_{2}^{2}-1}\right)}+\left(k_{1}^{2}+k_{2}^{2}\right) e^{\left(k_{1}+k_{2}\right) x+\left(\frac{k_{1}}{k_{1}^{2}-1}+\frac{k_{2}}{k_{2}^{2}-1}\right) t}}{\left(1+e^{\left(k_{1} x+\frac{k_{1} t}{k_{1}^{2}-1}\right)}+e^{\left(k_{2} x+\frac{k_{2} t}{k_{2}^{2}-1}\right)}\right)^{2}} \\
& V(x, t)=\frac{\left.2 k_{1}^{3} e^{\left(2 k_{1} x+\frac{2 k_{1} t}{k_{1}^{2}-1}\right)}+2 k_{2}^{3} e^{\left(2 k_{2} x+\frac{2 k_{2}}{k_{2}^{2}-1} t\right)}+\left(k_{1}-k_{2}\right)^{2}\left(k_{1}+k_{2}\right)\left(e^{\left(2 k_{1}+k_{2}\right) x+\left(\frac{2 k_{1}}{k_{1}^{2}-1}+\frac{k_{2}}{k_{1}^{2}-1}\right) t}+e^{\left(k_{2} x+\frac{k_{2} t}{k_{2}^{2}-1}\right)}\right)^{3}+e^{\left(k_{1}+2 k_{2}\right) x+\left(\frac{k_{1}}{k_{1}^{2}-1}+\frac{2 k_{2}}{k_{2}^{2}-1}\right) t}\right)}{(1+)^{3}}
\end{aligned}
$$

respectively,

For the three solitary wave solutions, we set the auxiliary function by

$$
f(x, t)=1+e^{k_{i} x+\frac{k_{1} t}{k_{1}^{2}-1}}+e^{k_{2} x+\frac{k_{2} t}{k_{2}^{2}-1}}+e^{k_{3} x+\frac{k_{3} t}{k_{3}^{2}-1}}
$$

proceeding as before, the three kink solutions and the three-soliton solutions can easily be found to be

$$
U=\frac{\sum_{i=1}^{3} k_{i}^{2} e^{k_{i} x+\frac{k_{i} t}{k_{i}^{2}}}+\sum_{\substack{i, j=1 \\ i>j}}^{3}\left(k_{i}-k_{j}\right)^{2} e^{\left(k_{i}+k_{j}\right) x+\left(\frac{k_{i}}{k_{i}^{2}-1}+\frac{k_{j}}{k_{j}^{2}-1}\right) t}}{\left(1+\sum_{i=1}^{3} e^{\left(k_{i} x+\frac{k_{i} t}{k_{i}^{2}-1}\right)}\right)^{2}}
$$




$$
\begin{gathered}
V=\frac{\sum_{i=1}^{3} k_{i}^{3} e^{k_{i} x+\frac{k_{i} t}{k_{i}^{2}-1}}+\sum_{\substack{i, j \\
i>j}}^{3}\left(k_{i}+k_{j}\right)\left(k_{i}-k_{j}\right)^{2} e^{\left(k_{i}+k_{j}\right) x+\left(\frac{k_{i}}{k_{i}^{2}-1}+\frac{k_{j}}{k_{j}^{2}-1}\right) t}}{\left(1+\sum_{i=1}^{3} e^{\left.k_{i} x+\frac{k_{i} t}{k_{i}^{2}-1}\right)^{2}}\right)} \\
-2\left(\sum_{i=1}^{3} k_{i} e^{k_{i} x+\frac{k_{i} t}{k_{i}^{2}-1}}\right)\left(\sum_{i=1}^{3} k_{i}^{2} e^{k_{i} x+\frac{k_{i} t}{k_{i}^{2}-1}}+\sum_{\substack{i, j=1 \\
i>j}}^{3}\left(k_{i}-k_{j}\right)^{2} e^{\left(k_{i}+k_{j}\right) x+\left(\frac{k_{i}}{k_{i}^{2}-1}+\frac{k_{j}}{k_{j}^{2}-1}\right) t}\right) \\
\left(1+\sum_{i=1}^{3} e^{k_{i} x+\frac{k_{i} t}{k_{i}^{2}-1}}\right)^{3}
\end{gathered}
$$

It is well known that the existence of three solitary wave solutions shows that the multiple solitary waves, including the $\mathrm{N}$-soliton solutionssolutions are obtainable (Hietaranta, 1987, Wazwaz, 2008)

\section{Other solutions: the hyperbolic functions methods}

In this section we will apply the hyperbolic functions approaches in order to determining some travelling wave solution. The schemes that will be used include:

\subsection{The tanh method}

In the tanh methodwe employ the use of the expression as used by Wazwaz, (2013)

$$
U(x, t)=\alpha+\beta \tanh (k x-\omega t) \quad \text { is allowed }
$$

as a solution of the system (1). To determine $\alpha, \beta$ and the wave speed $\omega$ we substitute (13) into (1) collect the coefficient of $\tanh ^{i}, i=0,1$, and equate it to zero to obtain

$$
2 \omega \beta-3 \alpha \omega \beta-k \beta=0
$$

Solving for $\omega$ we have

$$
\omega=\frac{k}{2-3 \alpha}
$$

where $\alpha \& \beta$ are assume to be free parameters.

This gives the solitary waves solutions of the system (1) as

$$
\begin{aligned}
& U(x, t)=\alpha+\beta \tanh \left(k x-\frac{k}{2-3 \alpha} t\right) \\
& V(x, t)=k \beta \sec h^{2}\left(k x-\frac{k}{2-3 \alpha} t\right)
\end{aligned}
$$

Substituting tanh forcoth in (13), and proceed in the same way as before we obtain the singular solutions.

$$
\left.\begin{array}{l}
U(x, t)=\alpha+\beta \operatorname{coth}\left(k x-\frac{k}{2-3 \alpha}\right) \\
V(x, t)=-k \beta \operatorname{csch}\left(k x-\frac{k}{2-3 \alpha}\right)
\end{array}\right\}
$$

The tan method

The tan method admits the used of the expression

$$
U(x, t)=\alpha+\beta \tan (k x-\omega t)
$$

as the solution of the system (1). To determined $\alpha, \beta$ and the wave speed $\omega$, we substitute (18) into (1), collect the coefficient of $\tan ^{i}, i=0,1$ and equate to zero we obtain

$-2 \omega \beta+3 \alpha \beta \omega+k \beta=0$

Solving for $\omega$ we obtain 


$$
\omega=\frac{k}{2-3 \alpha}
$$

where $\alpha$ is left as a free parameter.

This gives the solitary waves solutions of the system (1) by

$$
\begin{aligned}
& U(x, t)=\alpha+\beta \tan \left(k x-\frac{k}{2-3 \alpha} t\right) \\
& V=k \beta \sec ^{2}\left(k x-\frac{k}{2-3 \alpha} t\right)
\end{aligned}
$$

\subsection{The cot method}

Replacing tan by cot in (18) and proceeding as before we obtain the singular solutions

$$
\begin{aligned}
& U(x, t)=\alpha+\beta \cot \left(k x-\frac{k}{2-3 \alpha} t\right) \\
& V(x, t)=-k \beta \operatorname{cosec} c^{2}\left(k x-\frac{k}{2-3 \alpha} t\right)
\end{aligned}
$$

\subsection{The exp -trigonometric method}

In the exp -trigonometric we use the expression

$U(x, t)=e^{k x-\omega t}(\alpha \cos (k x-\omega t)+\beta \sin (k x-\omega t))$

as a solution of the system (1). To determine the wave speed, we substitute (19) into (1), collect the coefficients of

$e^{k x-\omega t} \cos (k x-\omega t), e^{k x-\omega t} \sin (k x-\omega t)$

and equate to zero to obtain

$$
\begin{aligned}
& k(\alpha+\beta)-2 \omega(\alpha+\beta)=0 \\
& k(\alpha-\beta)-2 \omega(\alpha-\beta)=0
\end{aligned}
$$

Solving for $\alpha, \beta$, and $\omega$ we obtain

$\omega=\frac{1}{2} k$

$\beta= \pm \alpha$

where $\alpha$ is chosen to be a free parameter,this gives the solitary waves solution to the system (1)

$$
\text { Bу } U(x, t)=\alpha e^{k x-\frac{1}{2} k t}\left(\cos \left(k x-\frac{1}{2} k t\right) \pm \sin \left(k x-\frac{1}{2} k t\right)\right)
$$

$$
V(x, t)=k \alpha e^{k x-\frac{1}{2} k t}\left(\cos \left(k x-\frac{1}{2} k t\right) \pm \sin \left(k x-\frac{1}{2} k t\right)\right)+k \alpha e^{k x-\frac{1}{2} k t}\left(-\sin \left(k x-\frac{1}{2} k t\right) \pm \cos \left(k x-\frac{1}{2} k t\right)\right)
$$

\section{Discussions}

The $(1+1)$ - dimensional Hirota Satsuma water- wave equation is investigated for multiple soliton solutions using different approach. Equation (1) models the unidirectional propagation of shallow water wave. The existence of the multiple soliton solutions and some travelling wave solutions indicates the variety of the application of the equation in engineering and science.

\section{References}

[1]. Ablowitz, M. J, H. Segur (1981),.Solitons and the inverse scattering transform (Philadelphia: SIAM,

[2]. A-M,.Wazwaz. (2013) Multiple soliton solutions and rational solutions for the (2+1)-dimensional dispersive long water-wave system OceanicEeng.60, 95-98

[3]. Freeman, N.C and Nimmo, J.J.C.(1983)The use of Backlund transformation in obtaining N-soliton solutions in Wronskian form. Phys. lett. A 95, 1-3.

[4]. Hereman, W. Nuseir, A. (1997), Symbolic method to construct exact solution of nonlinear partial differential equation Math, Comp, Simul., 43, 13-27

[5]. Hietarinta, J, (1987) A search for bilinear equation passing three-soliton condition I. KdV type bilinear equation, J. Math. Phys. 28, $1732-1742$

[6]. M.Y Adamu, E. sulaiman, (2012), On the generalized bilinear differential equations,J. Math 3 (4), 24-30

[7]. Ma.W. X (2003), diversity of exact solution to a restricted Baiti- panpinelli dispersive long way system, Physics,Letter. A310, 325333

[8]. R Hirota, 2004 The direct method in soliton theory, Cambridge University Press 
[9]. Su, T., Geng, X. G.and Yun-Ling, M.A. (2007),Wronskian form of N soliton solutions for the (2+1)-dimensional breaking soliton equations. Chin. Phy. Let. 24 (12) 305-307.

[10]. Wazwaz, A. (2008)Multiplesoliton solutions for Calogero-Bogoyavlensskii-Schiff, Jimbo- Miwa and YTSF equations, App. Math and comp. 203, , 592-597.

[11]. Wazwaz, A-M, (2007) Multiple soliton solution for the Boussinesq equation, Appl. Math.Comput. 192, 479-486

[12]. Xu, G. G. and Xu, Z. L. (2004),Symbolic computation of the Painleve test for nonlinear Differential equation, using maple. Comp. phys. Comm. 23, 65-75 